\title{
Association of Interferon Regulatory Factor-3 Gene Polymorphisms With Infection and Antiviral Efficacy of Chronic Hepatitis C Virus
}

\section{Xun-Jun Yang}

Wenzhou Medical College Second Affiliated Hospital: The Second Affiliated Hospital and Yuying Children's Hospital of Wenzhou Medical University

\section{Xiao-Ou Wang}

Wenzhou Medical College Second Affiliated Hospital: The Second Affiliated Hospital and Yuying Children's Hospital of Wenzhou Medical University

\section{Linna Liu}

Wenzhou Medical College Second Affiliated Hospital: The Second Affiliated Hospital and Yuying Children's Hospital of Wenzhou Medical University

\section{Yao Chen}

Wenzhou Medical University First Affiliated Hospital: The First Affiliated Hospital of Wenzhou Medical University

\section{Song-Dao Ye ( $\square$ yesd955022@163.com )}

Wenzhou Medical College Second Affiliated Hospital: The Second Affiliated Hospital and Yuying Children's Hospital of Wenzhou Medical University https://orcid.org/0000-0001-5984-1865

\section{Research}

Keywords: Hepatitis C virus, chronic infection, efficacy, genetic polymorphisms, interferon regulatory factor-3

Posted Date: August 30th, 2021

DOI: https://doi.org/10.21203/rs.3.rs-838971/v1

License: (c) (i) This work is licensed under a Creative Commons Attribution 4.0 International License. Read Full License 


\section{Association of Interferon Regulatory Factor-3 gene polymorphisms with infection and antiviral efficacy of chronic hepatitis $\mathrm{C}$ virus}

Running title: Interferon Regulatory Factor-3 gene polymorphisms with chronic hepatitis C virus

\section{Xunjun Yang ${ }^{1}$, Xiaoou Wang ${ }^{1}$, Linna Liu ${ }^{1}$, Yao Chen $^{2}$, Songdao Ye ${ }^{1 *}$}

${ }^{1}$ Department of Laboratory Medicine, The Second Affiliated Hospital and Yuying Children's Hospital of Wenzhou Medical University, Wenzhou, China

${ }^{2}$ Department of Pathologyine, The First Affiliated Hospital of Wenzhou Medical University, Wenzhou, China

*Corresponding author

Song-dao Ye, Department of Laboratory Medicine, The Second Affiliated Hospital and Yuying Children's Hospital of Wenzhou Medical University, Wenzhou, China.

Email: yesd955022@163.com 


\section{Abstract \\ background}

Hepatitis $\mathrm{C}$ virus (HCV) is a major public health concern in developing countries. Pathogenesis of hepatitis $\mathrm{C}$ infection is poorly understood. Previously, we found higher Interferon Regulatory Factor-3 (IRF-3) expression in HCV-infected patients. However, the effect of IRF-3 polymorphism on the incidence of HCV infection and antiviral efficacy has not been sufficiently evaluated.

\section{Methods}

We retrospectively enrolled 178 patients with chronic hepatitis $\mathrm{C}(\mathrm{CHC})$ and 82 matched healthy controls between 2016 and 2019 at the Second Hospital of Wenzhou Medical University, China. All patients received a standard dose of polyethylene glycol interferon + ribavirin (PR regimen), and were divided into the response, non-response, sustained virological response (SVR), and non-sustained virological response (NSVR) groups based on their HCV RNA levels. Gene polymorphisms were detected by DNA sequencing, and the plasma IRF-3 and IFN- $\beta$ levels were measured by ELISA to assess the impact of IRF-3 gene variations and contents on the risk of $\mathrm{HCV}$ infection and antiviral efficacy.

\section{Results}

Plasma contents of IRF-3 and IFN- $\beta$ were significantly different between the CHC and control groups, the response and non-response groups, and the SVR and non-SVR groups $(p<0.05)$. rs $2304206 \mathrm{C}>\mathrm{T}$ but not rs2304204A> $\mathrm{G}$ was associated with increased risk for $\mathrm{CHC}(\mathrm{OR}=2.35$ (95\% CI: 1.30 to 4.24), $p=0.004)$, and reduced antiviral efficacy between both response groups ( $\mathrm{OR}=1.86$ (95\% CI: 1.06 to 3.24), $p$ $=0.028)$ and SVR groups (OR=1.79 (95\% CI:1.05 to 3.06), $p=0.030)$. Haplotype GT type suffered negative consequence between the CHC and control groups, the responder and non-responder groups, and the SVR and non-SVR groups ( $p=0.004$, $0.028,0.030)$. The content of IFR-3 in CT genotype of rs 2304206 was higher than that in CC genotype.

Conclusion: The polymorphism of IFR-3 affects the plasma levels of IFR-3 and associated with HCV infection and interferon antiviral efficacy, where the T allele of rs2304206 may be a susceptibility factor for CHC and adversely impact interferon antiviral response.

Keywords: Hepatitis C virus; chronic infection; efficacy; genetic polymorphisms; interferon regulatory factor-3 


\section{Background}

Hepatitis $\mathrm{C}$ virus infection is one of the most common infectious diseases of global public health concern. According to the World Health Organization (WHO), the global prevalence of $\mathrm{HCV}$ infection is about $2.8 \%$, with about 185 million people infected worldwide [1]. There are more than 14.765 million HCV infections in China, which has the largest number of infections in the world[2]. HCV transmission route is known, but the onset of infection is insidious and chronicity is high, about $30 \%$ of chronic hepatitis $\mathrm{C}$ cases eventually develop cirrhosis and hepatocellular carcinoma (HCC), and there are more than 350,000 deaths every year[3, 4]. Chronicity after $\mathrm{HCV}$ infection results due to a combination of host immunity, genetic susceptibility and viral factors[5, 6]. Direct-acting antiviral (DAA) drugs for $\mathrm{CHC}$ have been approved for marketing in China, and these drugs have many advantages, but they are in the initial clinical stage, with little experience among different populations, and the cost is also high, so the pegylated interferon $\alpha+$ ribavirin (PR) regimen remains an important treatment for HCV infection[7]. Factors associated with the efficacy of PR regimens include viral factors (viral load, viral genotype and so on) $[8,9]$ and host factors (age, gender, single-nucleotide polymorphism (SNP), organism immune status, etc.) [10]. CHC is a polygenic infectious disease with a genetic predisposition. Many studies have shown that genetic polymorphisms such as IL-28B, CD209, CTLA4, ITPA, and MIC-1 are associated with CHC infection[11-15].

Interferon regulatory factors (IRFs) are a class of transcription factors that regulate gene expression of interferons (IFNs), and are closely related to immune response to pathogens, cytokine signaling and cell proliferation regulation. IRF-3 is an important member of the IRF family whose main function is to induce the production of type I IFN, IFN-stimulated genes (ISGs) and pro-inflammatory cytokines, which play a key role in the antiviral response[16]. The IRF-3 gene is located on the long arm of chromosome 19 between band 3 to band 4 of region 13 (19q13.3-q13.4). It has eight exons and seven introns, and the coding product consists of 427 amino acids with a relative molecular mass of approximately 55,000. Previous studies identified IRF-3 genetic variants that are associated with the risk of prostate cancer, systemic lupus erythematosus (SLE), chronic lymphocytic[17-19]. Since IRF-3 is an important host factor that may inhibit HCV, we investigated the relationship between IRF-3 and HCV infection by examining the genotype of IRF-3 gene polymorphism and the level of IRF-3 expression in patients with CHC and healthy controls. The goal of this study was to evaluate the association of IRF-3 with CHC and antiviral efficacy. To the best of our knowledge, this is the first study to report a relationship between IRF-3 genetic variants and $\mathrm{CHC}$.

\section{Methods \\ Study cohort \\ CHC group}

A total of $171 \mathrm{CHC}$ patients were recruited from the Infection Department of the Second Affiliated Hospital of Wenzhou Medical College between 2016 and 2019. This cohort fulfilled the diagnostic criteria of the Guidelines for Prevention and Treatment of Hepatitis C [7]. The inclusion criteria were as follows: age 18-70 years, 
HCV infection for $>6$ months or epidemiological history of six months, positive for anti-HCV and HCV RNA, histopathology of liver, HCV1b, no antiviral treatment and use of immunomodulators within three months, no absolute contraindication of PR treatment, can receive PR treatment, and signed the informed consent. The exclusion criteria were as follows: a combination of chronic hepatitis B and other hepatitis, alcoholic liver disease, drug-induced liver injury (DILI), autoimmune hepatitis, and other liver diseases, HIV infection, malignant tumors, patients with severe cerebrovascular disease, hematological disease, thyroid disease, diabetes, and incomplete data, which might affect efficacy and safety.

\section{Control group}

A total of 82 healthy subjects were enrolled from the Physical Examination Center at the Second Affiliated Hospital of Wenzhou Medical University as the control group. Exclusion criteria were as follows: accompanied by other viral hepatitis, such as chronic hepatitis B, alcoholic liver disease, DILI, autoimmune hepatitis, and other liver diseases; HIV infection; malignant tumor; severe cardiovascular and cerebrovascular diseases; hematological disease or thyroid disease; or diabetes. All study subjects were unrelated Han Chinese individuals from Zhejiang Province, China. This study was approved by the Medical Ethics Committee of the Second Affiliated Hospital of Wenzhou Medical University and conformed to the ethical guidelines of the Helsinki Declaration. Informed consent was obtained from all the enrolled patients.

\section{Determination of efficacy}

All patients were treated with pegylated interferon $\alpha(\mathrm{Peg}$ IFN $\alpha)$ combined with ribavirin (RBV) as follows: IFN- $\alpha-2 \mathrm{a}$ (subcutaneous injection, $180 \mu \mathrm{g}$ each time, once a week) and ribavirin (oral, 800-1000 mg/d), with a basic course of 48 weeks. HCV RNA was monitored before treatment and at 4, 12, 24 and 48 weeks of treatment to assess the viral response and adjust the dose accordingly. Efficacy determination and grouping [7]: according to the HCV RNA level at week 12 of treatment, the patients were classified as (1) virological response group: HCV RNA decreased $\geq 2 \mathrm{Log}$ at week 12 of treatment compared with that before treatment; (2) no virological response: HCV RNA decreased < 2Log at week 12 of treatment compared with that before treatment. According to the HCV RNA level at week 24 of follow-up after the end of treatment, the patients were classified as (1) Sustained virological response (SVR): HCV RNA was not measurable at week 24 after treatment; (2) Non-SVR: HCV RNA was measurable at week 24 after treatment.

\section{Sample collection}

About 4-5 mL of venous blood sample was collected from all fasting patients in EDTA-K2 in the morning. Thereafter, plasma and blood cells were separated and stored at $-80^{\circ} \mathrm{C}$. Plasma was used to detect IRF-3, IFN- $\beta$, HCV RNA and other parameters, while blood cells were used to extract DNA and analyze IRF-3 gene polymorphisms.

\section{Genomic DNA extraction and amplification}

Blood genomic DNA extraction kit (Shanghai Bioengineering Co., Ltd., SK8224) was used to extract human genomic DNA, according to the manufacturer's instructions. 
The amplification was performed using a polymerase chain reaction (PCR) amplification kit (SK2072; Sangon Biotech) in Veriti ® 96-well PCR instrument (Applied Biosystems Inc., Foster City, CA, USA). The PCR reaction conditions were as previously reported[20]. Briefly, pre-denaturation at $95^{\circ} \mathrm{C}$ for $3 \mathrm{~min}$, denaturation at $94^{\circ} \mathrm{C}$ for $30 \mathrm{~s}$, annealing at $58^{\circ} \mathrm{C}$ for $30 \mathrm{~s}$, extension at $72^{\circ} \mathrm{C}$ for $50 \mathrm{~s}$, total 35 cycles, repair extension at $72^{\circ} \mathrm{C}$ for $10 \mathrm{~min}$, storage at $4^{\circ} \mathrm{C}$. The primers were synthesized by Sangon Biotech as follows: rs2304204-F 5'-CTCCACTCAACTTGCGGTCTC-3', rs2304204-R 5'-CGGGT AGCTCTCTCAAACTCGA-3', rs2304206-F 5'-

GTTGGTTTTATTTCAAGAAGTCGAT-3', rs2304206-R 5'-GCTCCTTCCTTGCTCCACTTT-3'.

\section{Product purification and sequencing}

The PCR product purification and recovery kit (Shanghai Bioengineering Co., Ltd., SK1141) was used to purify the amplified products, and the NanoDrop 2000C UV spectrophotometer (Thermo, USA) was used for DNA quantitative analysis.

Sequencing was completed on a 3730XL sequencing instrument (ABI, USA), and the sequencing profiles were compared and analyzed using SeqMan software.

\section{Determination of plasma IRF-3 and IFN- $\beta$ levels}

The operation was completed using the double-antibody sandwich ELISA, according to the manufacturer's instructions (SuJingmei Biotechnology Co., Ltd.). The OD values of the samples and standards were detected with an Anthos 2010 enzyme standardizer (Shanghai Bosai Si Technology Co., Ltd.). The standard curve was drawn to calculate the IRF-3 and IFN- $\beta$ levels of the samples. If the OD values of the samples were beyond the upper limit of the standard curve, the determination was repeated with the appropriate dilution of the samples.

\section{Quantitative analysis of $\mathbf{H C V}$}

RNA was extracted by real-time quantitative fluorescence (FQ)-PCR according to the protocol of the kit (Piji Bioengineering Co., Ltd., Shenzhen, China), and detected using the ABI 7500 FQ-PCR instrument (Applied Biosystems Inc.). The normal reference ranges were defined as < 5.0 $\times 102$ copies/mL for HCV RNA.

\section{Statistical analysis}

All statistical analyses were performed using SPSS23.0. Pearson's chi-square test or Fisher's exact probability test was used for comparing the enumeration data between groups, mean and standard deviation was used to describe the normal distribution data, and t-test or variance analysis was used to compare the count data between groups. Median (M), 25th percentile (P25), and 75th percentile (P75) was used to represent the non-normal distribution data, and the rank-sum test was used for the inter-group comparison. Significant differences were noted in the genotype and allele frequency. Hardy-Weinberg equilibrium test, odds ratio (OR), and 95\% confidence interval (95\% $\mathrm{CI}$ ), linkage disequilibrium analysis, and haplotype construction were conducted using SHEsis (http://analy sis.biox.cn/myana lysis.php). The website of CHWE (https://www.genecalculators.net/pq-chwe-polypicker.html) was used for the analysis of heterozygosity and polymorphic information content (PIC) in all studied groups to determine whether the SNP was sufficiently polymorphic for conducting statistical analysis in the Chinese population. All tests were bilateral, and $p$-value $<0.05$ was 
considered statistically significant.

\section{Results}

\section{Comparison of baseline characteristics of the study subjects}

The CHC group included 99 males and 79 females, aged 18-74 (42.46 \pm 10.23$)$ years.

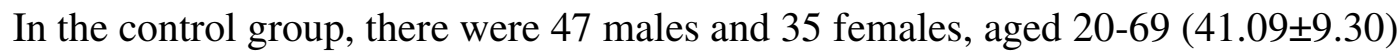
years. There was no difference in gender and age between the CHC group and healthy controls $(p>0.05)$. The statistical analyses showed significant difference in both IRF-3 and IFN- $\beta$ levels between the CHC group and control group, the response group and non-response group, and the SVR group and NSVR group (all $p<0.01$ ). The control group, response group and SVR group had higher levels of IRF-3 and IFN- $\beta$ (Table 1 and Fig1).

\section{IRF-3 genotypes and allele frequency distribution in the cohort}

Three genotypes, AA, AG and GG, were detected at rs2304204, and CC, CT and TT, were detected at rs2304206 of IRF-3 gene (Fig.2). By Hardy-Weinberg equilibrium test, each genotype of rs2304204 and rs2304206 were in accordance with the law of genetic equilibrium (all $p$ values $>0.05$ ). Heterozygosity and PIC calculation showed that both SNPs were sufficiently polymorphic for statistical analysis in the Chinese population (all $p$ values were $>0.3$ ). The genotype and allele frequencies of IRF-3 gene polymorphisms among the $\mathrm{CHC}$ and the healthy controls are shown in Table 2. There was no difference in rs2304204 genotype or allele between the CHC group and the control group $(p>0.05)$. However, there were significant differences in rs 2304206 genotype CC vs. CT (OR=2.87, 95\% CI: 1.47-5.60, $p=0.003$, after Bonferroni correction), and between the $\mathrm{C}$ allele and $\mathrm{T}$ allele (OR=2.35, 95\% CI: $1.30-4.24$, $p=0.008$, after Bonferroni correction) among the $\mathrm{CHC}$ and healthy controls. Gene linkage disequilibrium detection showed linkage disequilibrium between rs 2304204 and rs2304206 $\left(D^{\prime}=1.000, r 2=0.760\right)$. Haplotypes of two SNPs of IRF-3 gene (A-C, G-C, G-T) were constructed based on the LD results, in which the distribution of G-T type was statistically significant in the CHC and control groups $(p<0.05)$.

\section{Distribution of IRF-3 genotypes and allele frequencies in different antiviral efficacy groups}

There were no significant differences in genotype and allele frequency distribution of rs2304204 locus between the responder and non-responder groups, and between the SVR and non-SVR groups ( $p>0.05$ ). While rs2304206 locus genotype distribution showed significant difference between the SVR and non-SVR groups $(p=0.029)$, similar to the $\mathrm{C}$ allele distribution high proportion in the responder and SVR groups than the non-responder and non-SVR groups $(p=0.028,0.030$, respectively). The differences in haplotype G-T were also significantly different between the responder and non-responder groups, the SVR and non-SVR groups $(p=0.028,0.030$, respectively). The results are shown in Table 3.

\section{Comparison of plasma IRF-3 and IFN- $\beta$ levels between different genotypes}

To analyze the reasons for the correlation between rs2304206 and CHC, we compared the differences in IRF-3 levels among different genotypes. The levels of IRF-3 among each genotype at locus rs2304206 in all study subjects were as follows: CC genotype $(\mathrm{n}=187, \mathrm{M}=237.44, \mathrm{P} 25=137.67, \mathrm{P} 75=484.59)$, CT genotype $(\mathrm{n}=69, \mathrm{M}=$ 
169.16, P25 = 80.33, P75 = 263.38) and TT genotype $(\mathrm{n}=4, \mathrm{M}=152.43, \mathrm{P} 25=110.10$, P75=303.97). Rank-sum test of multiple independent samples showed statistically significant differences in IRF-3 levels between rs2304206 genotypes ( $p=0.005)$. Among them, CC genotype was significantly higher than CT and TT genotypes ( $p<$ 0.01, after Bonferroni correction, as seen in Fig.3). There were no differences in IRF-3 levels between genotypes at the rs2304204 (as shown in Fig.4). Similarly, plasma IFN- $\beta$ levels between different genotypes at the rs2304204 and rs2304206 loci in all study subjects were not statistically significant (all $p>0.05$, data not shown).

\section{Discussion}

IRF-3 is an important IFN-regulated transcription factor that plays an important role in immune response and immune regulation after viral infection[21, 22]. Several viruses such as hepatitis B virus, HCV, and HIV can bind to cell surface receptors, leading to rapid activation of IRF-3 in the cytoplasm, initiating ISG transcription, promoting cytokine production, and preventing viral replication and spread [23]. When the virus enters the host cell and starts replication, the viral component is also able to inhibit IRF-3 expression, phosphorylation and nuclear translocation through multiple pathways, which is one of the ways by which the virus evades the intrinsic immune response [24].

The current study showed that the levels of plasma IRF-3 was negatively correlated with $\mathrm{HCV}$ infection and positively correlated with antiviral efficacy, suggesting an association between plasma IRF-3 levels and HCV infection and prognosis. However, IRF-3, as a protein that is induced under multiple stresses, differs significantly between individuals and at different stages of disease progression, requiring multiple measurements and dynamic observations to be of clinical value. The specific mechanism of IRF-3 in HCV infection and the factors determining IRF-3 levels are unclear, and these issues could be clarified by the study of IRF-3 polymorphisms at the molecular level. Several studies found an association between IRF-3 gene polymorphisms and various diseases. Zhang [25] et al. reported that polymorphisms in the IRF-3 gene codon 427 increased susceptibility to esophageal carcinogenesis. Garifulin[26] et al. revealed that polymorphisms in IRF-3 may affect the expression of IFN- $\beta$, thus affecting the antiviral immune response. Akahoshi[18] et al. discovered a significant association between SNPs in the promoter region of IRF-3 gene and resistance to SLE.

The hotspot SNPs of IRF-3 gene were rs2304204 and rs2304206 loci. Most studies concluded that rs2304204 does not affect IRF-3 protein expression and is not significantly associated with the disease[27-29]. The present study showed that the genotype and allele frequency distribution of the rs2304204 locus were not associated with CHC and plasma levels of IRF-3 or IFN- $\beta$. However, in terms of the other locus rs2304206, Zhang[19] et al. found that genetic polymorphism was associated with susceptibility and prognosis of chronic lymphocytic leukemia (CLL) in the Han Chinese population. Bhawna[17] et al. reported that the rs2304206 locus polymorphism was associated with the risk of prostate cancer in Jamaican population. However, rs2304204 and rs2304206 loci polymorphisms of IRF-3 gene were not associated with genetic susceptibility to HBV infection in Chinese Han population 
[30]. This study found that genotypes and allele frequency distribution of rs2304206 differed significantly between the $\mathrm{CHC}$ and control groups, the response and non-response groups, and the SVR and non-SVR groups, and the level of IRF-3 was also affected. Thus, we hypothesized that the rs 2304206 locus polymorphism might be associated with CHC susceptibility and IFN antiviral efficacy by affecting IRF-3 expression levels. This study also revealed a linkage disequilibrium between the rs2304204 and rs2304206 loci $\left(D^{\prime}=1.000, r 2=0.760\right)$, with statistically significant differences in the distribution of haplotype G-T types between the CHC and control groups, the responder and non-responder groups, and the SVR and non-SVR groups $(p<0.05)$.

This study had some limitations. The study subjects were all from Zhejiang, China, the sample size was small, and the group of virus natural clearers were not included in this study since they did not meet the statistical requirements. These factors may have led to selection bias and the role of IRF-3 in HCV natural clearance could not be explored. Therefore, expanding the selection area and sample size, and increasing the number of viral natural clearance groups are needed in future research.

\title{
Conclusion
}

The IRF-3 gene rs2304206 polymorphism affects the plasma level of this protein and is associated with HCV infection and interferon antiviral efficacy, where the T allele of rs2304206 and rs2304204/rs2304206 haplotype G-T may be susceptibility factors for HCV infection and unfavorable for interferon response.

\begin{abstract}
Abbreviations
HCV: hepatitis C virus; CHC: chronic hepatitis C; SVR: sustained virological response; NSVR: non-sustained virological response; IRF-3: Interferon Regulatory Factor-3; HCC: hepatocellular carcinoma; IRFs: Interferon regulatory factors; IFNs: interferons; ISGs: IFN-stimulated genes; SLE: systemic lupus erythematosus; DILI: drug-induced liver injury; CLL: chronic lymphocytic leukemia.
\end{abstract}

\section{Acknowledgements}

We thank Dr. Wei Lin and other doctors in the Department of Infectious Diseases, Second Hospital of Wenzhou Medical University for their support and assistance in the collection of chronic hepatitis $\mathrm{C}$ cases, clinical diagnosis and efficacy determination.

\section{Authors' contributions}

XJY and XOWcarried out the study, participated in collecting data, and drafted the manuscript. LNL and YC provided the suggestive advices for this research and took part in revised the manuscript. SDYe conceived the study and revised the paper. All authors read and approved the final manuscript.

\section{Funding}

This study was Supported by the Zhejiang Medical and Health Science and 
Technology Plan Project, No. 2016KYB191

\section{Availability of data and materials}

The data used to support the findings of this study are mainly included within the article, and the underlying data are available from the corresponding author upon request.

\section{Ethics approval and consent to participate}

Ethics approval is not applicable. Informed consent was obtained from each participant.

\section{Consent for publication}

Not applicable.

\section{Competing interests}

The authors declare that they have no competing interests.

\section{Author details}

1Department of Laboratory Medicine, The Second Affiliated Hospital and Yuying Children's Hospital of Wenzhou Medical University, Wenzhou, China. 2 Department of Pathologyine, The First Affiliated Hospital of Wenzhou Medical University, Wenzhou, China 


\section{REFERENCES}

1. WHO Guidelines Approved by the Guidelines Review Committee. In: Guidelines for the Care and Treatment of Persons Diagnosed with Chronic Hepatitis C Virus Infection. edn. Geneva: World Health Organization@ World Health Organization 2018.; 2018.

2. Gower E, Estes C, Blach S et al. Global epidemiology and genotype distribution of the hepatitis $\mathrm{C}$ virus infection. Journal of hepatology. 2014; 61(1 Suppl):S45-57.

3. Mohd Hanafiah K, Groeger J, Flaxman AD et al. Global epidemiology of hepatitis C virus infection: new estimates of age-specific antibody to HCV seroprevalence. Hepatology (Baltimore, Md). 2013; 57(4):1333-1342.

4. Lemoine M, Nayagam S, Thursz M Viral hepatitis in resource-limited countries and access to antiviral therapies: current and future challenges. Future virology. 2013; 8(4):371-380.

5. Heim $\mathrm{MH}$, Thimme R Innate and adaptive immune responses in HCV infections. Journal of hepatology. 2014; 61(1 Suppl):S14-25.

6. Irshad M, Mankotia DS, Irshad K An insight into the diagnosis and pathogenesis of hepatitis C virus infection. World journal of gastroenterology. 2013; 19(44):7896-7909.

7. Chinese Society of Hepatology; Chinese Medical Association; Wei L. [The guideline of prevention and treatment for hepatitis C: a 2015 update]. Zhonghua Gan Zang Bing Za Zhi 2015; 23: 906-923

8. EASL Clinical Practice Guidelines: management of hepatitis C virus infection. Journal of hepatology. 2014; 60(2):392-420.

9. Brady RR, Ventham NT, Roberts DM et al. Open transversus abdominis plane block and analgesic requirements in patients following right hemicolectomy. Annals of the Royal College of Surgeons of England. 2012; 94(5):327-330.

10. Li LF, Shi KQ, Lin YQ et al. Factors associated with efficacy of pegylated interferon- $\alpha$ plus ribavirin for chronic hepatitis C after renal transplantation. Gene. 2014; 544(2):101-106.

11. Naeemi H, Aslam R, Raza SM et al. Distribution of IL28B and IL10 polymorphisms as genetic predictors of treatment response in Pakistani HCV genotype 3 patients. Archives of virology. 2018; 163(4):997-1008.

12. Zupin L, Polesello V, Alberi G et al. CD209 promoter polymorphisms associate with HCV infection and pegylated-interferon plus ribavirin treatment response. Molecular immunology. 2016; 76:49-54.

13. Enciso-Vargas M, Ruíz-Madrigal B, Muñoz-Valle JF et al. Association of -319 C/T and +49 A/G polymorphisms of CTLA-4 gene in patients with hepatitis C virus infection. Medicina clinica. 2018; 150(7):251-256.

14. Liu Z, Wang S, Qi W et al. The relationship between ITPA rs1127354 polymorphisms and efficacy of antiviral treatment in Northeast Chinese CHC patients. Medicine. 2017; 96(29):e7554.

15. Yang XJ, Wang XO, Chen $\mathrm{Y}$ et al. Associations of content and gene polymorphism of macrophage inhibitory factor-1 and chronic hepatitis $\mathrm{C}$ virus infection. World journal of gastroenterology. 2020; 26(41):6378-6390.

16. Yang $\mathrm{S}$, Zhan $\mathrm{Y}$, Zhou $\mathrm{Y}$ et al. Interferon regulatory factor 3 is a key regulation factor for inducing the expression of SAMHD1 in antiviral innate immunity. Scientific reports. 2016; 6:29665. 
17. Dubey B, Jackson M, Zeigler-Johnson C et al. Interactive effect of TLR SNPs and exposure to sexually transmitted infections on prostate cancer risk in Jamaican men. The Prostate. 2020; 80(15):1365-1372.

18. Akahoshi M, Nakashima H, Sadanaga A et al. Promoter polymorphisms in the IRF3 gene confer protection against systemic lupus erythematosus. Lupus. 2008; 17(6):568-574.

19. Zhang ZX, Shen CF, Shou LH et al. IRF-3 gene polymorphisms are associated with the susceptibility to and the survival in chronic lymphocytic leukemia and could also serve as an auxiliary index. Leukemia \& lymphoma. 2017; 58(3):646-654.

20. Ye S, Chen Y, Lou X et al. Association of macrophage inhibitory factor -1 polymorphisms with antiviral efficacy of type $1 \mathrm{~b}$ chronic hepatitis C. Molecular and cellular biochemistry. 2021.

21. Chattopadhyay S, Kuzmanovic T, Zhang Y et al. Ubiquitination of the Transcription Factor IRF-3 Activates RIPA, the Apoptotic Pathway that Protects Mice from Viral Pathogenesis. Immunity. 2016; 44(5):1151-1161.

22. Bedard KM, Wang ML, Proll SC et al. Isoflavone agonists of IRF-3 dependent signaling have antiviral activity against RNA viruses. Journal of virology. 2012; 86(13):7334-7344.

23. Vilasco M, Larrea E, Vitour D et al. The protein kinase IKKepsilon can inhibit HCV expression independently of IFN and its own expression is downregulated in HCV-infected livers. Hepatology (Baltimore, Md). 2006; 44(6):1635-1647.

24. Homma T, Ishibashi D, Nakagaki T et al. Persistent prion infection disturbs the function of Oct-1, resulting in the down-regulation of murine interferon regulatory factor-3. Scientific reports. 2014; 4:6006.

25. Zhang CF, Cao BW, Lu ZM et al. [Relationship between polymorphism of IRF-3 gene codon 427 and esophageal cancer in Anyang population of China]. Beijing da xue xue bao Yi xue ban= Journal of Peking University Health sciences. 2004; 36(4):345-347.

26. Garifulin O, Qi Z, Shen H et al. Irf3 polymorphism alters induction of interferon beta in response to Listeria monocytogenes infection. PLoS genetics. 2007; 3(9):1587-1597.

27. Mosayyebi S, Sarac BE, Akel Bilgic H et al. The Genetic Variants of Interferon Regulatory Factor-3 in Children with Asthma. Journal of interferon \& cytokine research : the official journal of the International Society for Interferon and Cytokine Research. 2020; 40(12):570-577.

28. Lin JD, Yang SF, Wang YH et al. Associations of gene polymorphisms in interferon-alpha signature-related genes with autoimmune thyroid diseases. Clinical endocrinology. 2019; 91(6):860-868.

29. Real LM, Caruz A, Rivero-Juarez A et al. A polymorphism linked to RRAS, SCAF1, IRF3 and BCL2L12 genes is associated with cirrhosis in hepatitis C virus carriers. Liver international : official journal of the International Association for the Study of the Liver. 2014; 34(4):558-566.

30. Yan F, Gao YF, Lv F et al. No association between IRF3 polymorphism and susceptibility to hepatitis B virus infection in Chinese patients. World journal of gastroenterology. 2012; 18(4):388-392. 


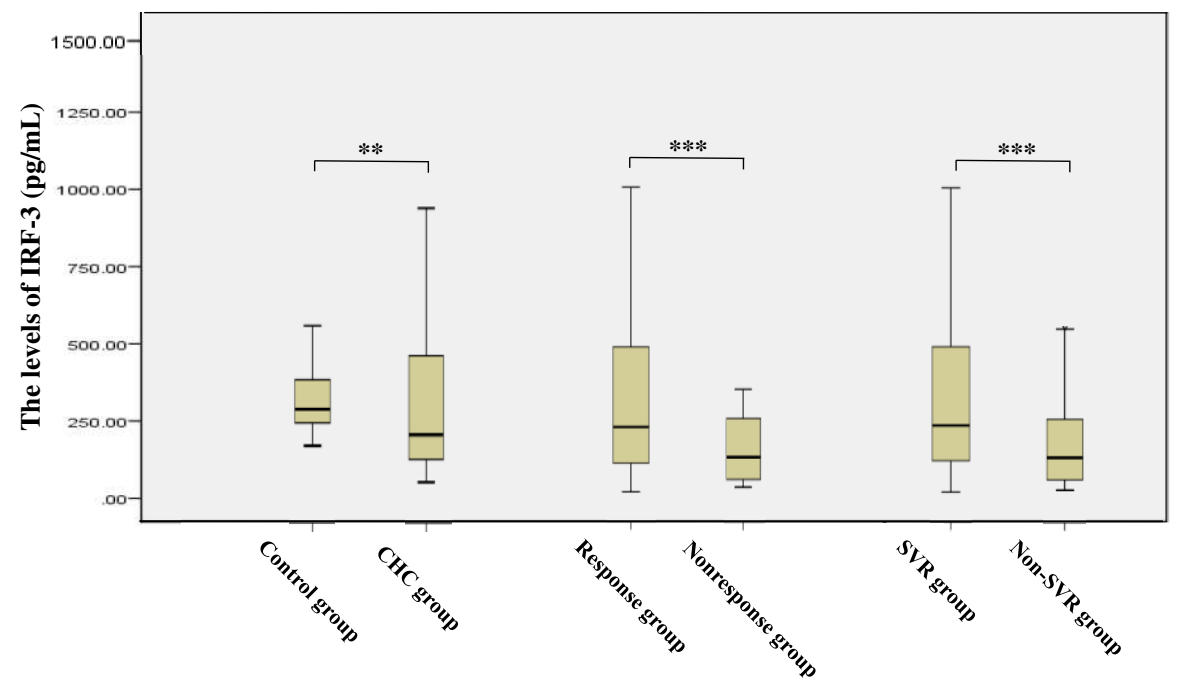

Fig.1 Comparison of IRF-3 level among different groups. The level of IRF-3 in the control group was significantly higher than in CHC group $(p<0.01)$. Similarly, significant difference was observed between response group and non-response group $(p<0.001)$. The same result was SVR group have higher level of IRF-3 than non-SVR group $(p<0.001)(* *$ mean $p<0.01, * * *$ mean $p<0.001$ by Mann - Whitney $\mathrm{U}$ test).

Table 1 Comparison of baseline characteristics between chronic hepatitis $\mathbf{C}$ and control groups

\begin{tabular}{cccc}
\hline Parameter & Control group $\mathrm{n}=82$ & CHC group $\mathrm{n}=178$ & P value \\
\hline Gender, M/F & $47 / 35$ & $99 / 79$ & 0.80 \\
Age & $41.1 \pm 9.3$ & $42.5 \pm 10.2$ & 0.30 \\
IRF-3 in $\mathrm{pg} / \mathrm{ml}, \mathrm{M} / \mathrm{P} 25 / \mathrm{P} 75$ & $252.5 / 208.7 / 362.1$ & $170.3 / 191.4 / 27.4$ & $\mathbf{0 . 0 0 4}$ \\
IFN- $\beta$ in $\mathrm{pg} / \mathrm{ml}, \mathrm{M} / \mathrm{P} 25 / \mathrm{P} 75$ & $5.9 / 4.9 / 8.9$ & $3.6 / 2.3 / 6.0$ & $\mathbf{0 . 0 0 1}$ \\
\hline
\end{tabular}

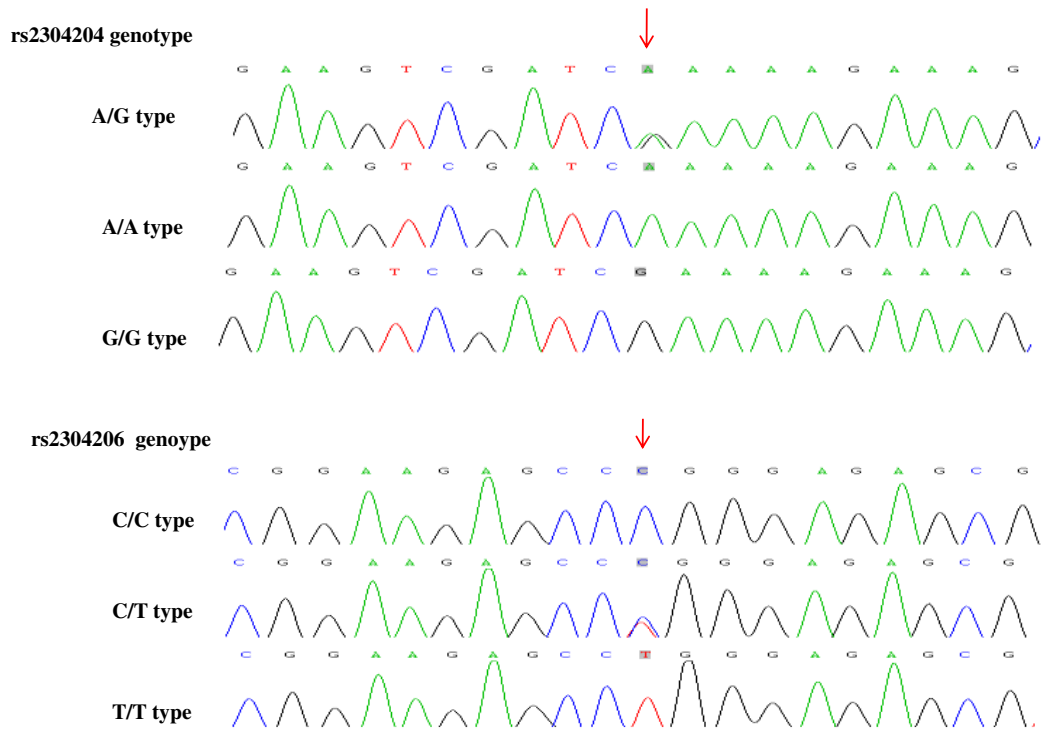

Fig.2 Sequencing map of IRF-3 gene polymorphic loci. 
Table 2. Genotypes and allele distribution in subjects

\begin{tabular}{|c|c|c|c|c|}
\hline Genotype & $\begin{array}{l}\text { Control group } \\
n=82(\%)\end{array}$ & $\begin{array}{l}\text { CHC group } \\
\mathrm{n}=178(\%)\end{array}$ & $p$ value & OR $(95 \% C I)$ \\
\hline \multicolumn{5}{|c|}{ rs2304204 gene } \\
\hline AA & $58(72.0)$ & $109(61.2)$ & & \\
\hline $\mathrm{AG}$ & $23(26.8)$ & $59(33.1)$ & 0.15 & \\
\hline GG & $1(1.2)$ & $10(5.6)$ & & \\
\hline A & $139(84.8)$ & $277(77.8)$ & 1 & Reference \\
\hline $\mathrm{G}$ & $25(15.2)$ & $79(22.2)$ & 0.066 & $1.59(0.97-2.60)$ \\
\hline \multicolumn{5}{|c|}{ rs2304206 gene } \\
\hline $\mathrm{CC}$ & $68(82.9)$ & $113(63.5)$ & & \\
\hline $\mathrm{CT}$ & $13(15.9)$ & $62(34.8)$ & $0.006 *$ & \\
\hline $\mathrm{TT}$ & $1(1.2)$ & $3(1.7)$ & & \\
\hline $\mathrm{C}$ & 149 (90.9) & $288(80.9)$ & 1 & Reference \\
\hline $\mathrm{T}$ & $15(9.1)$ & $68(19.1)$ & $0.004 *$ & $2.35(1.30-4.24)$ \\
\hline \multicolumn{5}{|l|}{ Haplotype } \\
\hline A-C & $139.00(84.8)$ & $277(77.8)$ & 0.066 & $0.631(0.385-1.033)$ \\
\hline $\mathrm{G}-\mathrm{C}$ & $10.00(6.1)$ & $11.00(3.1)$ & 0.106 & $0.491(0.204-1.180)$ \\
\hline G-T & $15.00(9.1)$ & $68.00(19.1)$ & $0.004 *$ & $2.345(1.296-4.244)$ \\
\hline
\end{tabular}

* $p$ values adjusted by Bonferroni correction was still significant. 
Table 3 Genotype and allele distribution of different therapeutic groups in patients with CHC

\begin{tabular}{|c|c|c|c|c|c|c|c|c|}
\hline \multirow[b]{2}{*}{ Genotype } & \multicolumn{4}{|c|}{ Evaluation of therapeutic effect at 12 weeks } & \multicolumn{4}{|c|}{ Follow-up to 24 weeks for efficacy judgment } \\
\hline & $\begin{array}{l}\text { Response group } \\
\quad(\mathrm{n}=129)\end{array}$ & $\begin{array}{l}\text { Non-response } \\
\qquad(\mathrm{n}=49)\end{array}$ & $p$ value & OR $(95 \% C I)$ & $\begin{array}{l}\text { SVR group } \\
(\mathrm{n}=129)\end{array}$ & $\begin{array}{c}\text { Non-SVR } \\
(n=49)\end{array}$ & $p$ value & OR $(95 \% C I)$ \\
\hline \multicolumn{9}{|l|}{ rs2304204 } \\
\hline A & $207(0.802)$ & $70(0.714)$ & 1 & Reference & $173(0.808)$ & $104(0.732)$ & 1 & Reference \\
\hline G & $51(0.198)$ & $28(0.286)$ & 0.074 & $0.616(0.361-1.051)$ & $41(0.192)$ & $38(0.268)$ & 0.091 & $0.649(0.392-1.074)$ \\
\hline AA & $84(0.651)$ & $25(0.510)$ & & & $70(0.654)$ & $39(0.549)$ & & \\
\hline $\mathrm{AG}$ & $39(0.302)$ & $20(0.408)$ & 0.209 & & $33(0.308)$ & $26(0.366)$ & 0.236 & \\
\hline GG & $6(0.047)$ & $4(0.082)$ & & & $4(0.037)$ & $6(0.085)$ & & \\
\hline HWE & $P=0.594$ & $P=0.901$ & & & $P=0.964$ & $P=0.579$ & & \\
\hline \multicolumn{9}{|l|}{ rs2304206 } \\
\hline $\mathrm{C}$ & $216(0.837)$ & $72(0.735)$ & & & $181(0.846)$ & $107(0.754)$ & 1 & Reference \\
\hline $\mathrm{T}$ & $42(0.163)$ & $26(0.265)$ & 0.028 & $1.86(1.06-3.24)$ & $33(0.154)$ & $35(0.246)$ & 0.030 & $1.79(1.05-3.06)$ \\
\hline $\mathrm{CC}$ & $88(0.682)$ & $25(0.510)$ & & & $74(0.692)$ & $39(0.549)$ & & \\
\hline $\mathrm{CT}$ & $40(0.310)$ & $22(0.449)$ & 0.052 & & $33(0.308)$ & $29(0.408)$ & 0.029 & \\
\hline TT & $1(0.008)$ & $2(0.041)$ & & & $0(0.000)$ & $3(0.042)$ & & \\
\hline HWE & $P=0.594$ & $P=0.957$ & & & $P=0.524$ & $P=0.401$ & & \\
\hline \multicolumn{9}{|l|}{ Haplotype } \\
\hline A-C & $207.00(0.802)$ & $70.00(0.714)$ & 0.074 & $0.616(0.361 \sim 1.051)$ & $173.00(0.808)$ & $104.00(0.732)$ & 0.091 & $0.649(0392-1.074)$ \\
\hline G-C & $9.00(0.035)$ & $2.00(0.020)$ & 0.481 & $0.576(0.122 \sim 2.716)$ & $8.00(0.037)$ & $3.00(0.021)$ & 0.385 & $0.556(0.154-2.131)$ \\
\hline G-T & $42.00(0.163)$ & $26.00(0.265)$ & 0.028 & $1.857(1.064 \sim 3.242)$ & $33.00(0.154)$ & $35.00(0.246)$ & 0.030 & $1.794(1.054-3.055)$ \\
\hline
\end{tabular}




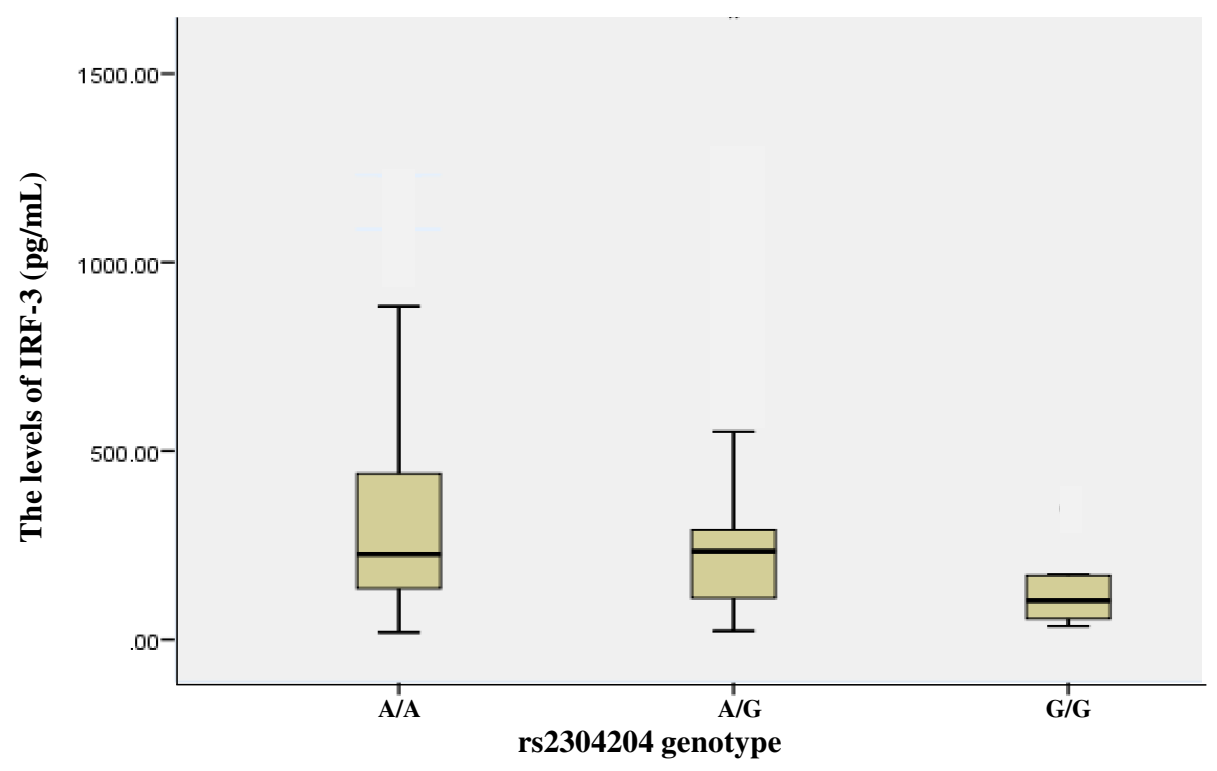

Fig. 3 Comparison in IRF-3 level among different genotypes at rs2304204 locus. The IRF-3 levels of various genotypes at rs2304204 locus were as follows:( $\mathrm{n}=171, \mathrm{M}=227.23, \mathrm{P} 25=135.82$, $\mathrm{P} 75=439.86)$ for genotype AA, $(\mathrm{n}=78, \mathrm{M}=233.88 .40, \mathrm{P} 25=108.93, \mathrm{P} 75=302.01)$ for genotype AG, and $(\mathrm{n}=11, \mathrm{M}=103.02, \mathrm{P} 25=54.36, \mathrm{P} 75=173.55)$ for genotype $\mathrm{GG}$. The rank-sum tests of various independent samples showed that no statistically significant difference was observed in the IRF-3 level for different genotypes $(p=0.055)$

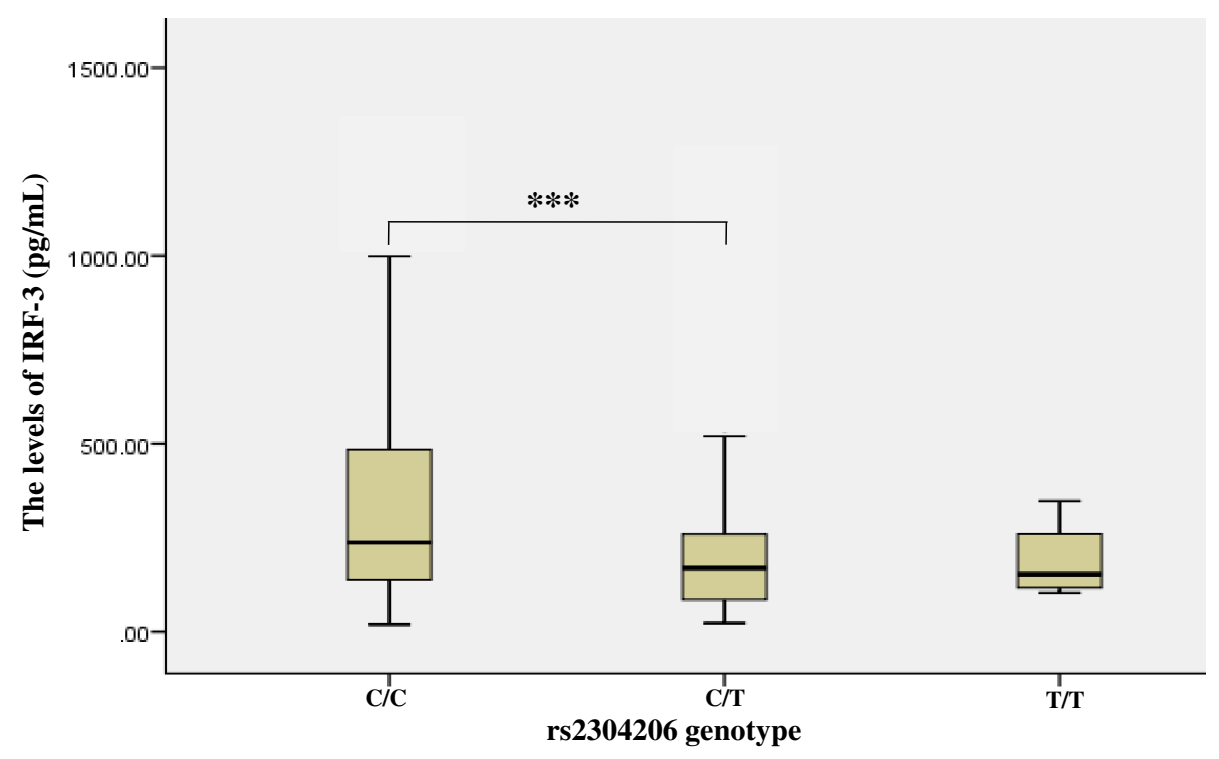

Fig.4 Comparison in IRF-3 level among different genotypes at rs2304206 locus. The IRF-3 levels of various genotypes at rs2304206 locus were as follows: $(n=187, M=237.44, P 25=137.67$, $\mathrm{P} 75=484.59)$ for genotype $\mathrm{CC},(\mathrm{n}=69, \mathrm{M}=169.16, \mathrm{P} 25=80.33, \mathrm{P} 75=263.38)$ for genotype $\mathrm{CT}$, and $\mathrm{n}=4, \mathrm{M}=152.43, \mathrm{P} 25=110.10, \mathrm{P} 75=303.97)$ for genotype TT. The rank-sum tests of various independent samples showed that statistically significant difference was observed in IRF-3 level for different genotypes $(p=0.005)$, and CC genotype was significantly higher than CT genotype $(* * *$ mean $p<0.001)$. 